\title{
Ion Beam Processing for Sample Preparation of Hybrid Materials with Strongly Differing Mechanical Properties
}

\author{
Khemais Barienti ${ }^{1}\left[\right.$ C Christoph Kahra ${ }^{1} \cdot$ Sebastian Herbst ${ }^{1} \cdot$ Florian Nürnberger $^{1} \cdot$ Hans Jürgen Maier $^{1}$
}

Received: 9 September 2019 / Revised: 22 November 2019 / Accepted: 17 December 2019 / Published online: 22 January 2020

(c) The Author(s) 2020

\begin{abstract}
For studies of the interface zone of metallic composites with significantly differing mechanical properties across the interface, high-quality sample preparation is paramount. In particular, the analysis of the composition or geometric characteristics of intermetallic phases close to the interface requires a preparation specifically adapted to the actual sample. In the present study, the combination of ion beam processing and a conventional metallographic preparation routine is investigated. It is shown that by utilizing a suitable combination of metallographic and ion beam processing routines, the interface zone of metallic composites can be prepared specifically targeted to a given analytical task. Especially for material combinations with largely differing mechanical properties of the base materials, ion beam processing can greatly improve the sample preparation quality.
\end{abstract}

Keywords Interfaces $\cdot$ Intermetallics $\cdot$ Aluminum alloys $\cdot$ Steels $\cdot$ Ion beam treatment

\section{Introduction}

The requirements placed on metallic components have increased steadily in recent years. Especially in the automotive and aerospace industry, increasing functionality and performance combined with decreasing component masses are demanded. However, reducing the mass while simultaneously increasing the loads poses a challenge due to the limited specific strength of the materials.

One possible approach is the use of metallic hybrid materials, for example by producing shafts of aluminum and steel by friction welding. In this case, the component properties can be tailored to specifically fit the local requirements. For instance, such hybrid components can combine the lightweight construction potential of aluminum with the high strength and wear resistance of steel. The prerequisite for their usability is a high strength of the joining zone of the two materials. At the interface of steel and aluminum, intermetallic phases can form that limit the bond strength [9]. Since processing affects the phase evolution at the interface,

Khemais Barienti

barienti@iw.uni-hannover.de; k.barienti@googlemail.com

$1 \quad$ Institut für Werkstoffkunde (Materials Science), Leibniz Universität Hannover, An der Universität 2, 30823 Garbsen, Germany it is mandatory to characterize the process-microstructure-property relationship in detail.

To study the interface zone and the intermetallic phases of friction-welded steel-aluminum components precisely, metallographic microsections are needed. Typically, a series of grinding and polishing operations are required to produce the specimens. During these processes, the two metals respond differently to the preparation due to their specific mechanical properties, further aggravating the mechanical sample preparation [12]. If preparation procedures are not carefully adapted, a distinct step is formed in the joining zone between aluminum and steel, which can make subsequent examination by scanning electron microscopy (SEM) extremely difficult [3]. Thus, the objective of the present study was to develop a preparation strategy for steel-aluminum bond zones that minimizes this step height by combining mechanical preparation steps with ion beam processing. The sample preparation prior to ion beam treatment was based on a topography-optimized specimen processing technique developed by Herbst et al. [8]. They show that especially during mechanical polishing the step height in the intermetallic bond zone between the harder and the softer material can increase. To address this effect, ion beam processing was carried out on both polished and ground samples. The ion beam processing was based on the work of Besserer et al. [2], where it was employed to prepare samples for the investigation of ductile damage in deep-drawn 
Table 1 Chemical composition (wt.\%) of the materials used as determined by optical emission spectroscopy; only elements with a mass fraction above $0.05 \%$ are listed

\begin{tabular}{llllllllll}
\hline Material & $\mathrm{Al}$ & $\mathrm{C}$ & $\mathrm{Cr}$ & $\mathrm{Cu}$ & $\mathrm{Fe}$ & $\mathrm{Mg}$ & $\mathrm{Mn}$ & $\mathrm{Ni}$ & $\mathrm{Si}$ \\
\hline 20MnCr5 & $\ldots$ & 0.20 & 1.08 & 0.27 & $\mathrm{Bal}$ & $\ldots$ & 1.23 & 0.11 & 0.24 \\
EN AW-6082 & $\mathrm{Bal}$ & $\ldots$ & 0.14 & 0.06 & 0.27 & 0.66 & 0.57 & $\ldots$ & 1.06 \\
\hline
\end{tabular}

Table 2 Hardness of $20 \mathrm{MnCr} 5$ (1.7147) and EN AW-6082 (AlMgSi1) materials for 30-mm-diameter round specimens

\begin{tabular}{lc}
\hline & $\begin{array}{l}\text { Hardness, } \\
\text { HV10 }\end{array}$ \\
\hline 20MnCr5 & 190 \\
EN AW-6082 & 87 \\
after T4 & \\
\hline
\end{tabular}

steels. In their study, Besserer et al. analyzed the effects of ion beam intensity, processing time and angle of incidence on surface topography. Their results served as the starting point for the parameters used during ion processing in the present study.

\section{Experimental}

An advanced process chain for bulk-forming of joined hybrid components has been established in the previous research [10]. In the present study, the same frictionwelded steel-aluminum hybrid component consisting of the case-hardening steel $20 \mathrm{MnCr} 5$ and the aluminum alloy $\mathrm{EN}$ AW-6082 (AlMgSi1) was employed exemplarily to examine the sample preparation for metallic composites with significantly differing mechanical properties.

Following the friction welding, a T4 heat treatment (solution heat-treated and naturally aged) of the component was carried out to increase the thickness of formed intermetallic phase layers in the bonding zone and to improve the mechanical properties of the part. The chemical compositions and hardness values of the base materials are shown in Tables 1 and 2 .

For the metallographic preparation and the subsequent ion beam processing, specimens with dimensions of $5 \mathrm{~mm} \times 2.6 \mathrm{~mm} \times 2 \mathrm{~mm}$ were machined from the bond zone by electrical discharge machining. The samples were subjected to a total of four grinding operations using $\mathrm{SiC}$ abrasive paper up to a final paper grade of 4000. Since preliminary investigations revealed that the specimen alignment during the individual grinding steps was particularly decisive for the surface quality, the bond zone was re-positioned after each grinding step as shown in Fig. 1. After grinding, an additional mechanical vibration polishing for one hour was carried out for selected samples with a VibroMet 2 vibration polisher (Buehler) using an oxidic polishing suspension (EPOSIL M11, ATM). The subsequent test series for the ion beam processing were carried out on both initial states, hereinafter referred to as ground (ground to grade 4000) and vibropolished (ground to grade 4000 and vibration-polished for one hour).

The ion beam processing was carried out with a MetEtch 683 unit (Gatan). The effects of the three possible ion beam processing variants ion polishing, slope cutting and ion etching on the surface roughness and the step height of

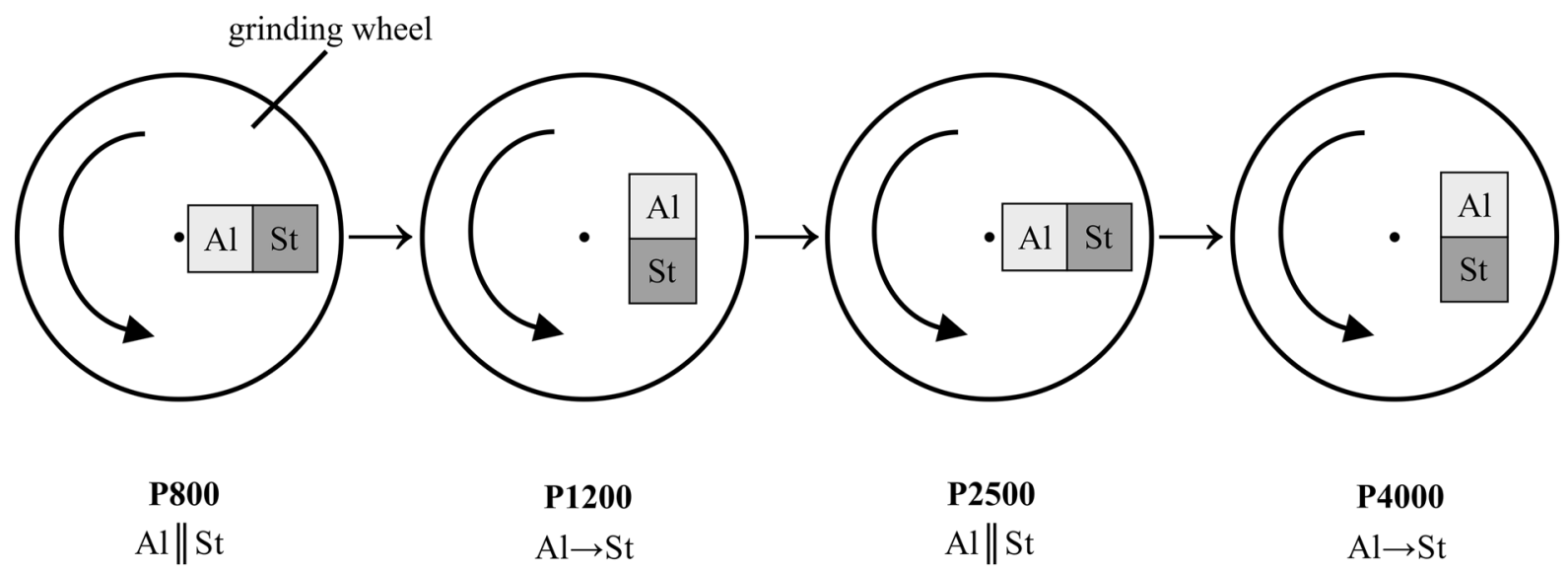

Fig. 1 Representation of the sample positioning used (AlllSt and ALSt) during the four successive grinding operations for metallographic preparation 
Table 3 Examined preparation routines for metallographic pretreatment and subsequent ion beam processing

\begin{tabular}{|c|c|c|c|c|}
\hline \multicolumn{5}{|c|}{$1^{\text {st }}$ grinding } \\
\hline \multicolumn{5}{|c|}{ er $150 \mathrm{U} / \mathrm{min}$} \\
\hline \multicolumn{5}{|c|}{$2^{\text {nd }}$ grinding } \\
\hline \multicolumn{5}{|c|}{ er $150 \mathrm{U} / \mathrm{min}$} \\
\hline \multicolumn{5}{|c|}{$3^{\text {nd }}$ grinding } \\
\hline \multicolumn{5}{|c|}{ A1\|St P2500 paper } \\
\hline \multicolumn{3}{|c|}{$\begin{array}{l}4^{\text {th }} \text { grinding } \\
\text { "ground" }\end{array}$} & \multicolumn{2}{|c|}{$\begin{array}{l}4^{\text {th }} \text { grinding } \\
\text { "vibropolished" }\end{array}$} \\
\hline \multirow[t]{2}{*}{ Al-St } & \multicolumn{2}{|c|}{ P4000 paper $150 \mathrm{U} / \mathrm{min}$} & \multicolumn{2}{|c|}{$\begin{array}{c}\text { Al-St } \mathrm{P} 4000 \text { paper } 150 \mathrm{U} / \mathrm{min} \\
\text { medium: Water } \\
\text { +vibrationpolishing }\end{array}$} \\
\hline & & & duratios & $0 \mathrm{~min}$ medium: $\mathrm{M} 11$ \\
\hline \multicolumn{2}{|c|}{$\begin{array}{l}\text { Ion-treatment } \\
\text { "ion polishing" }\end{array}$} & \multicolumn{2}{|c|}{$\begin{array}{l}\text { Ion-treatment } \\
\text { "slope cut" }\end{array}$} & $\begin{array}{l}\text { Ion-treatment } \\
\text { "ion etching" }\end{array}$ \\
\hline $\begin{array}{r}4 \mathrm{keV} 18 \\
\mathrm{Al}\end{array}$ & Angle: $88^{\circ}$ & \multicolumn{2}{|c|}{ Angle: $30^{\circ}$} & $\begin{array}{c}4 \mathrm{keV} 90 \min \mathrm{FZ} \perp \mathrm{WM} \\
\text { Angle: } 0^{\circ}\end{array}$ \\
\hline
\end{tabular}

the bonding zone were evaluated. Ion etching is often used for the metallographic preparation of surfaces and allows microstructural contrasting of the treated surfaces [5]. Ion beam slope cutting offers a method for artifact-free preparation. The sputtering takes place up to a sharp boundary line, which is given by the projection of a tungsten aperture edge. Between the ablated and non-ablated areas, an embankment area develops, which exposes a representative sectional area through the material. It is free of mechanical deformations and is not selectively skimmed by grazing ions $[1,7]$. As the ions only graze the sample surface during ion polishing, it results in only low removal rates [11]. However, the fact that the ions do not hit the sample surface directly also reduces the selective attack of the sample surface [6].

For processing, the ion beam unit was operated with an acceleration voltage of $4 \mathrm{keV}$, which represents a good compromise between the atomization yield and the load or service life of the ion source [13]. The preparation duration was set to $90 \mathrm{~min}$ or $180 \mathrm{~min}$. The named processing variants differ by the geometric angle between the sample's surface normal and the ion beam. The investigated alignments were $88^{\circ}$ (ion polishing), $60^{\circ}$ (slope cutting) and $0^{\circ}$ (ion etching). In Table 3, the employed mechanical preparation and ion beam treatments are summarized.
The subsequent characterization of the ion-processed surfaces was carried out in two steps. First, a confocal laser scanning microscope (type VK 9710, Keyence) was used to measure the samples' topography. On each of the samples examined, several three-dimensional depth images were taken on a $200-\mu \mathrm{m}^{2}$ surface. The topographic information was used to determine the final sample quality as described by Herbst et al. [8] using the step parameters $H_{10}, H_{20}$ and $H_{100}$. As depicted in Fig. 2, these three indicators provide information on the mean step height in a certain distance to the bond zone. To determine these key indicators, a total of nine profiles were distributed over three depth images per sample. The high-resolution depth images were also employed for determining the roughness parameters $R_{z}$ and $R_{a}$. For this purpose, twelve square areal integrals were specified on each image and then statistically evaluated. Since the sample surfaces created by slope cutting have a very small area of interest, only one profile line per specimen could be analyzed. For the same reason, the roughness values were not determined in this case. Following the geometric surface characterization, further analysis was performed by scanning electron microscopy (SEM) with a SUPRA 55 VP (Zeiss).

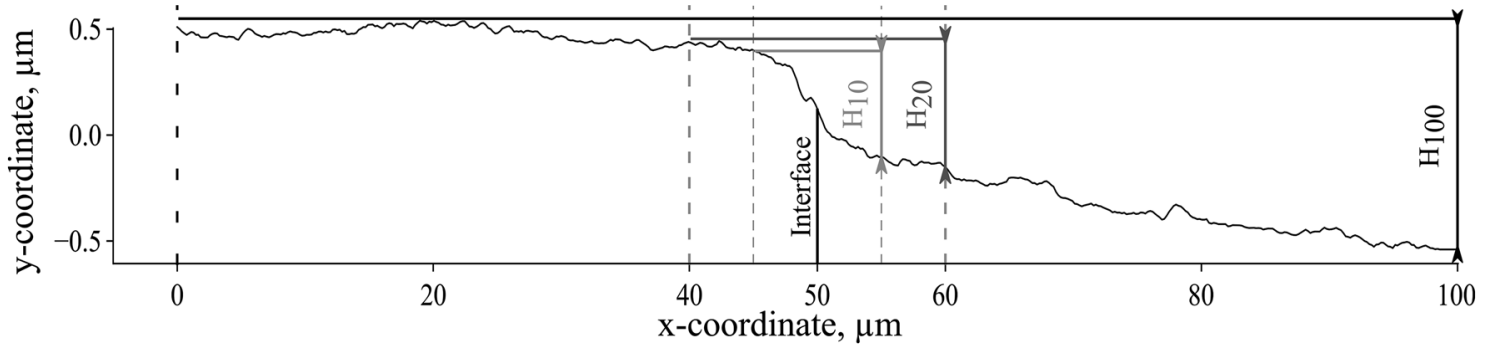

Fig. 2 Definition of the height parameters $H_{10}, H_{20}$ and $H_{100}$ used for the evaluation of the generated step height 


\section{Results and Discussion}

\section{Influence of the Mechanical Preparation}

Figure 3 depicts representative line-profiles measured by confocal laser scanning microscopy of the respective preparation routines for both initial states. A specific influence of the individual angle of incidence during ion processing that also varies with the different initial states (ground or vibropolished) is evident. This effect becomes particularly clear by comparing the respective step parameters $H_{10}$, $H_{20}$ and $H_{100}$ (Fig. 4) as well as the roughness development (Fig. 5). Generally, a preceding vibration polishing already results in significantly lower roughness parameters $R_{\mathrm{z}}$ and $R_{a}$ for the investigated surface areas. This tendency is retained after ion processing, so that the initially polished specimens always feature the lowest roughness. The recorded profile characteristics (Fig. 3) reflect this as well. However, the observed tendencies cannot be transferred
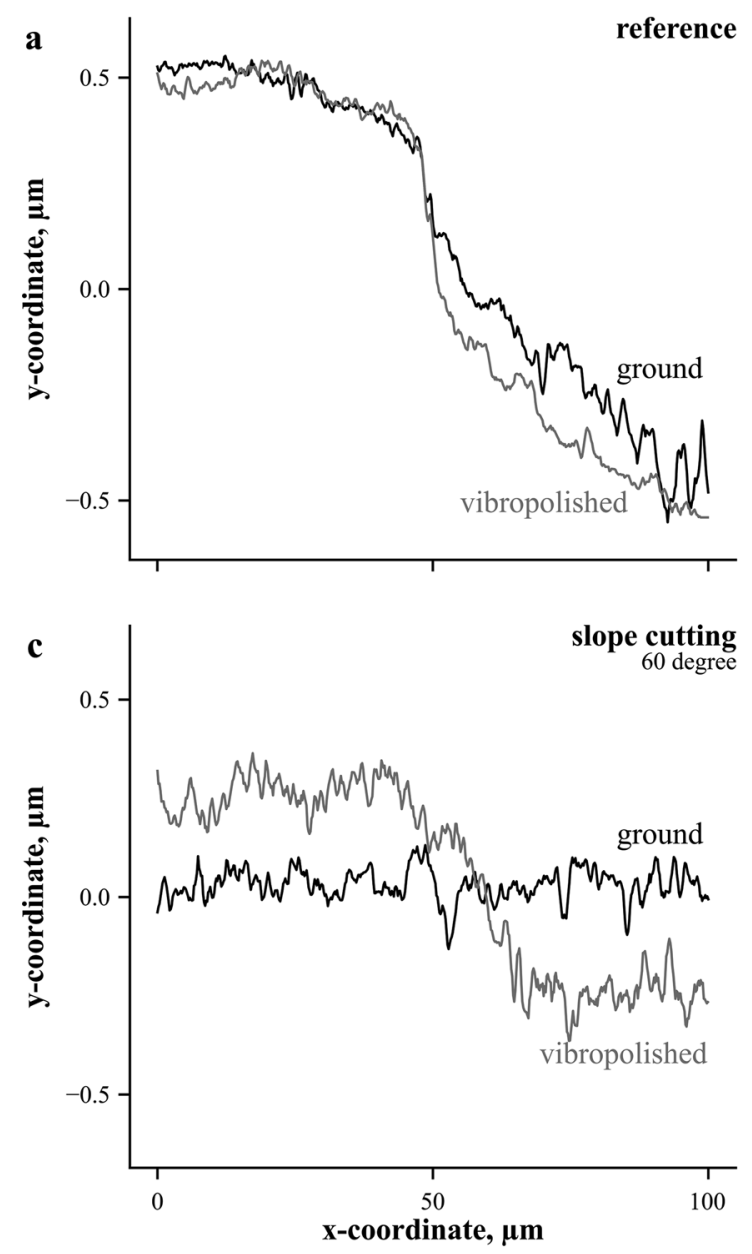

to the measured step heights (Fig. 4). Considering the three step height parameters of all preparation routines, the initial condition ground always resulted in a lower step formation despite poorer surface roughness. This phenomenon can be attributed to the fact that in all the experiments the initial state ground already exhibits a lower step height. The observed difference may be due to the selectively preferred material removal on the softer base material, in this case aluminum. Loose particles within the polishing suspension can accumulate there and preferably remove material at the interface.

\section{Ion Beam Polishing}

An ionic treatment of the specimen under a sharp angle of incidence $\left(88^{\circ}\right.$, ion polishing) for $180 \mathrm{~min}$ results only in a slight change of the general specimen topography. Both the overall shape of the recorded profile (Fig. 3-ion-polished) and the respective step height parameters show only minimal changes. However, roughness peaks are removed
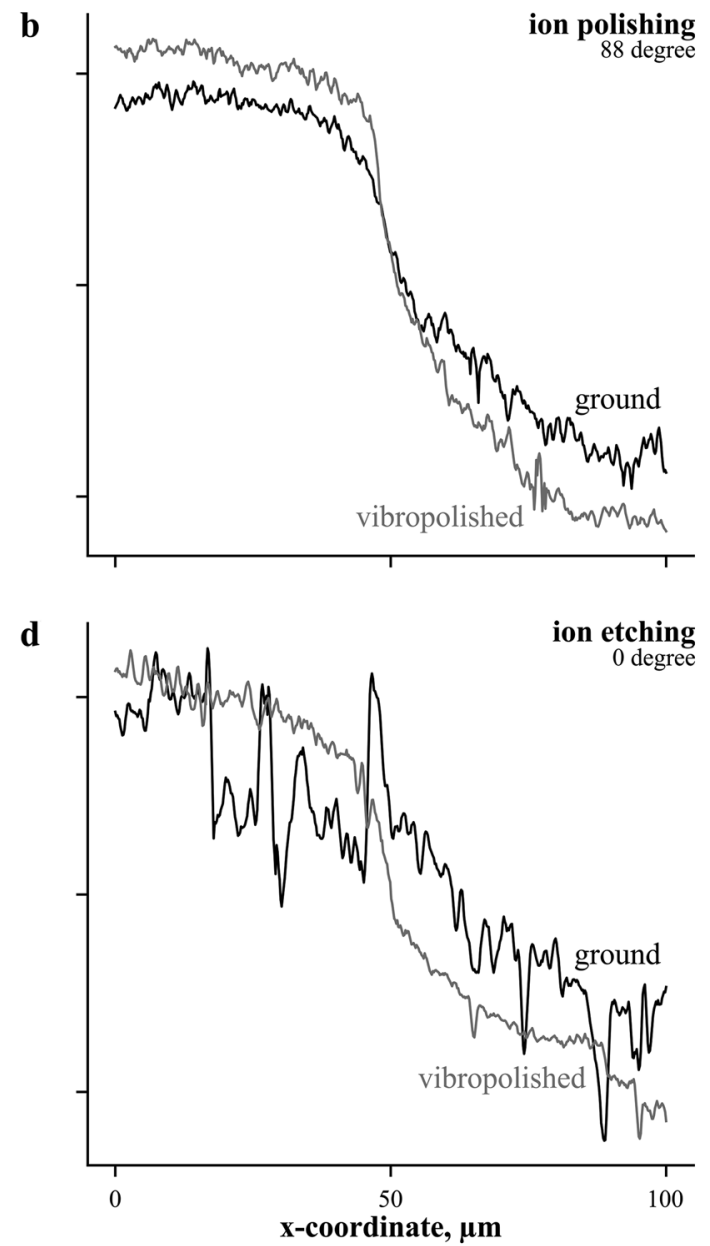

Fig. 3 Height profiles of the reference (a), ground and vibropolished ion-polished, (b), slope cutting (c) and ion-etched (d) samples 

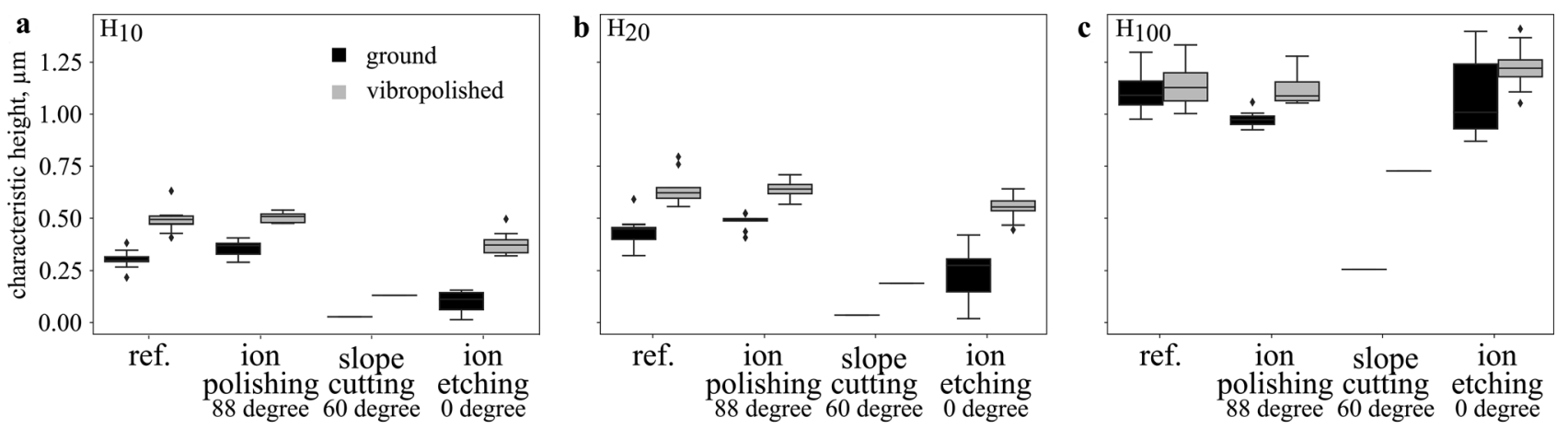

Fig. 4 Measured height profile characteristics $H_{10}(\mathbf{a}), H_{20}(\mathbf{b})$ and $H_{100}(\mathbf{c})$ for the separate reference (ref.) states and the corresponding investigated operating points during ion processing

a

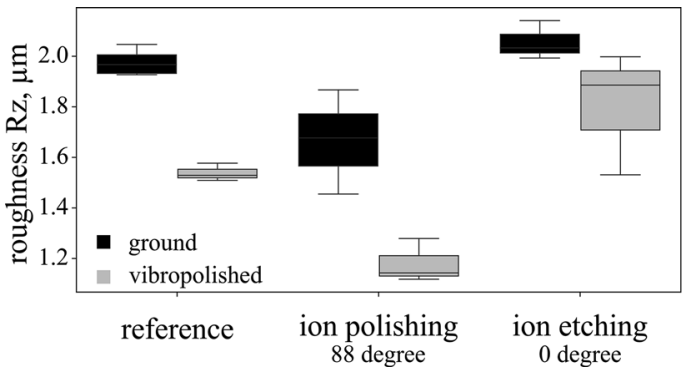

b

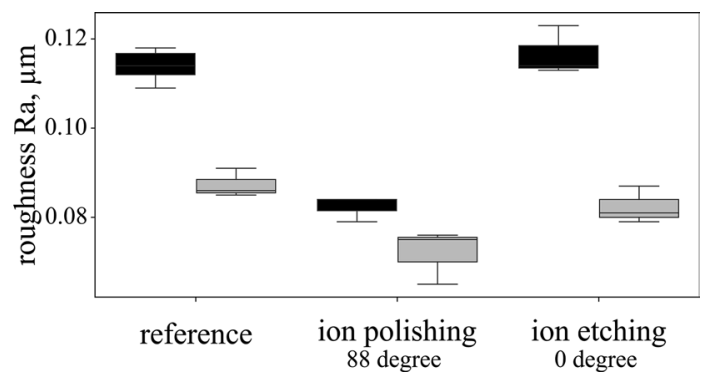

Fig. 5 Measured roughness coefficients $R_{z}(\mathbf{a})$ and $R_{a}$ (b) for the respective reference states as well as the corresponding operating points ion polishing and ion etching

over the entire sample area. In particular, this results in a measurable reduction of the mean roughness $R_{a}$ of up to $12.9 \%$ for previously vibratory polished samples and of up to $26.5 \%$ for those without mechanical polishing. The observed homogenization of the surface is due to the deviating ablation rate as a function of the respective local angle of incidence of the beam [4]. The angle of incidence becomes particularly steep at protruding irregularities and roughness peaks, so that a higher amount of energy is transferred locally, and these areas are preferentially removed. The rougher the surface to be treated, the more peaks of roughness are removed on average. This consequently means that the initially rougher condition (ground) is subjected to a noticeable stronger polishing effect. However, the image of an ion-polished sample in Fig. 6b (ion polishing) demonstrates that the surface was not homogeneous after ion polishing, and a formation of grooves can be observed along the processing direction. These grooves, typically named "comet tails," occur in particular at pores or inclusions and are the product of the unidirectional polishing treatment. This phenomenon can be observed, especially in the previously vibration-polished samples. Vibration polishing can cause pre-loosening of inclusions and thus result in a larger number of pores causing the observed tailing.

\section{Ion Beam Slope Cutting}

The use of a so-called slope cut under an angle of incidence of $60^{\circ}$ offers the possibility to expose a very small area of the compound zone. According to Hauffe [7], this method is suitable for realizing almost artifact-free surfaces. Especially with an alignment of the cutting edge perpendicular to the bond zone, the slope cut proves to be an excellent tool for a step-less preparation of the interface. This is reflected in the consistently lowest parameters $H_{10}, H_{20}$, and $H_{100}$ along the slope. The direct step height $\left(H_{10}\right)$ could be reduced by $90 \%$ compared to the initial ground state and by $73 \%$ compared to the initially vibration-polished state. From the SEM images in Fig. 6c (slope cutting), it can be seen that the respective bond partners were ablated at a different rate during preparation. About twice as much material was removed from the steel (left) than from the aluminum part (right). This phenomenon is due to the oxidation layer on top of the surface of the aluminum. It has a higher resistance to the ion treatment and has to be removed prior to the removal of the aluminum matrix underneath. Thus, the removal of the aluminum matrix sets in later compared to steel. After $90 \mathrm{~min}$ of ion treatment, an edge perpendicular to the joining zone with a width of approximately $4 \mu \mathrm{m}$ was prepared almost artifact- and step-free. As already noticed for ion-polished 

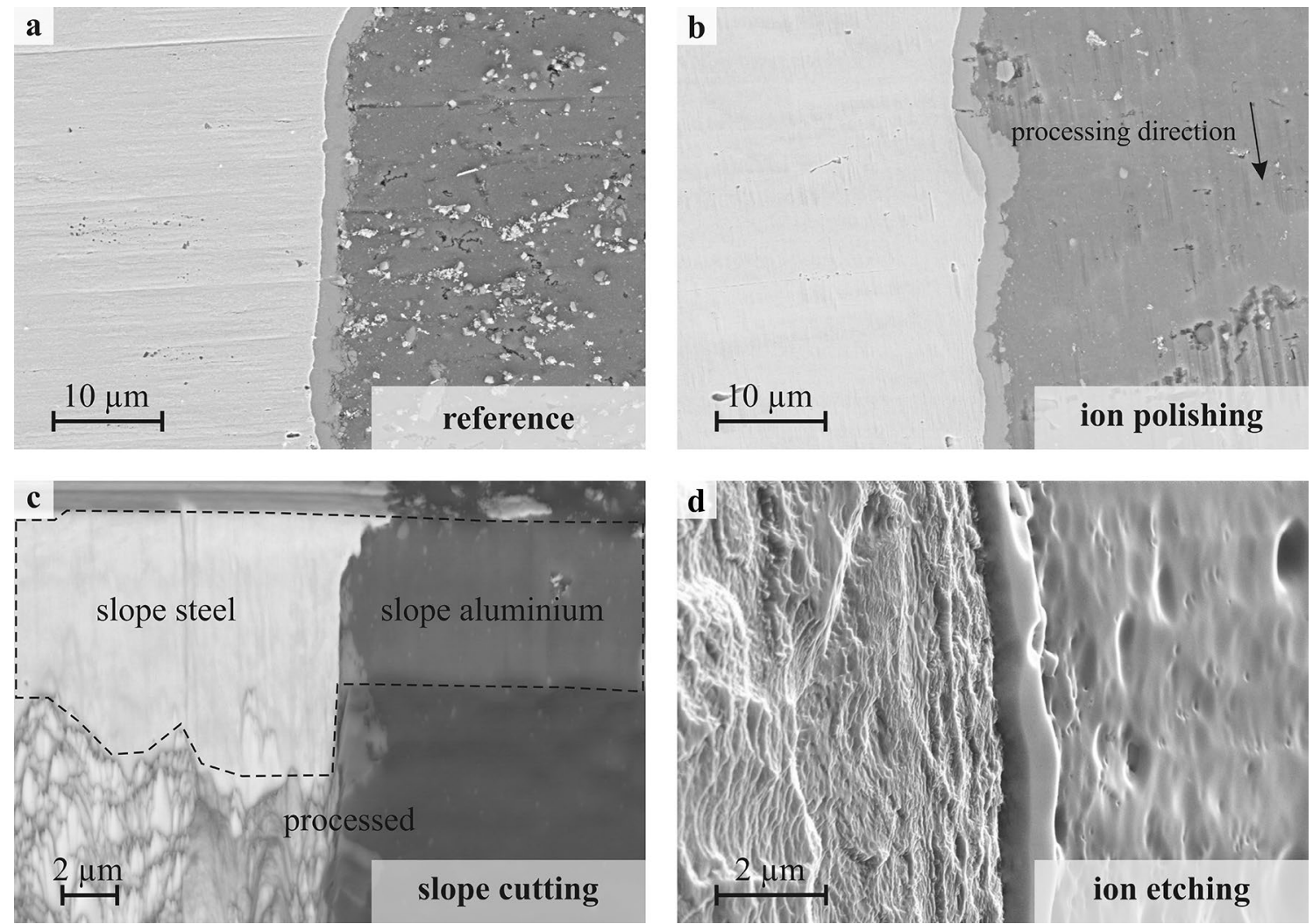

Fig. 6 SEM images of the differently prepared sample surfaces: initial state vibropolished (a), vibropolished+ion-polished (b), vibropolished + slope cutting $(\mathbf{c})$ and ground + ion-etched $(\mathbf{d})$

samples, slight grooves can be seen along the slope cut surface. These grooves can be recognized equally in both matrix materials.

\section{Ion Beam Etching}

An additional preparation method of the intermetallic phase in the interface is ion etching, i.e., with a treatment angle of $0^{\circ}$. This setting results in a lower material removal rate of the intermetallic phase compared to the surrounding base materials. Thus, the intermetallic phase appears protruded in SEM images (Fig. 6d). In addition, material removal during ion etching is much more inhomogeneous compared to ion polishing. This is in line with similar results demonstrated in the work of Besserer et al. [2]. Both observed phenomena are also reflected in the recorded profile lines in Fig. $3 \mathrm{~d}$ as well as the associated characteristic parameters (Fig. 5). The previously vibration-polished sample does not exhibit the phenomenon of protruding the intermetallic phase (Fig. 3d) and on average has a lower variation in the geometrical characteristics. On both samples, the material removal took place unevenly within the respective matrix. According to Gräf [4], this is due to the change in material removal rates for given crystallographic planes. This results in both ion-etched states showing a slightly higher roughness than the corresponding initial states.

\section{Conclusions}

The influence of ion beam processing on the preparation quality of steel-aluminum hybrid materials that feature strongly differing mechanical properties of the base materials was investigated. For this purpose, the effects of different operation points during ionic processing on the resulting geometrical shape of the bond zone and the roughness properties were investigated using confocal laser microscopy and SEM. It could be shown that the interface zone of hybrid materials can be specifically prepared using ion processing in combination with a careful selection of a preceding mechanical preparation:

- A combination of grinding, vibratory polishing and ion polishing, at an angle of incidence of $88^{\circ}$ produces a sample surface with minimal roughness.

- Performing a slope cut perpendicular to the composite zone at an angle of incidence of $60^{\circ}$ on previously ground 
specimens frees up a small sample area that is virtually artifact- and step-free.

- Ion etching on previously ground specimens results in a material-specific uneven ablation, so that the intermetallic phase protrudes and can be examined independent from the surrounding matrix materials and parallel to the bond plane.

Acknowledgements Open Access funding provided by Projekt DEAL. Financial support of this study by Deutsche Forschungsgemeinschaft (Grant 252662854) is gratefully acknowledged.

Open Access This article is licensed under a Creative Commons Attribution 4.0 International License, which permits use, sharing, adaptation, distribution and reproduction in any medium or format, as long as you give appropriate credit to the original author(s) and the source, provide a link to the Creative Commons licence, and indicate if changes were made. The images or other third party material in this article are included in the article's Creative Commons licence, unless indicated otherwise in a credit line to the material. If material is not included in the article's Creative Commons licence and your intended use is not permitted by statutory regulation or exceeds the permitted use, you will need to obtain permission directly from the copyright holder. To view a copy of this licence, visit http://creativecommons.org/licenses/by/4.0/.

\section{References}

1. R. Behrisch, Festkörperzerstäubung durch Ionenbeschuss, in Ergebnisse der exakten naturwissenschaften, ed. by S. Flügge, F. Trendelenburg (Springer, Berlin, 1964), pp. 295-443

2. H.-B. Besserer, G. Gerstein, A. Dalinger, L. Jablonik, D. Rodman, F. Nürnberger, Ion beam processing in the sample preparation for the analysis of ductile damage in deep drawing steels. Pract. Metallogr. 53(4), 221-236 (2016)

3. P.J. Goodhew, J. Humphreys, R. Beanland, Electron Microscopy and Analysis, 3rd edn. (Taylor \& Francis, London, 2001), pp. $170-174$
4. I. Gräf, Ion etching - state of the art and perspectives for contrasting the microstructure of ceramic and metallic materials. Part I: development and physics in ionetching. Pract. Metallogr. 35(5), 235-254 (1998)

5. I. Gräf, Ion etching - state of the art and perspectives for contrasting the microstructure of ceramic and metallic materials Part III: ionetching in metallography. Pract. Metallogr. 35(7), 359-383 (1998)

6. W. Hauffe, Elektronenmikroskopische Untersuchungen zur Entwicklung der Abbaustruktur auf Metalloberflächen bei Beschuß mit $10 \mathrm{keV}$-Argonionen, Dissertation, Technische Universität Dresden Dresden, 1971

7. W. Hauffe, Production of microstructures by ion beam sputtering, in Sputtering by Particle Bombardment III Characteristics of Sputtered Particles Technical Applications, ed. by R. Behrisch, K. Wittmaack (Springer, Berlin, 1991), pp. 305-338

8. S. Herbst, L. Jablonik, G. Gerstein, F. Nürnberger, F.-W. Bach, The preparation of metallic joinings to optimise their topography. Pract. Metallogr. 50(7), 491-500 (2013)

9. S. Herbst, H. Aengeneyndt, H.J. Maier, F. Nürnberger, Microstructure and mechanical properties of friction welded steel-aluminum hybrid components after T6 heat treatment. Mater. Sci. Eng. A 696, 33-41 (2017). https://doi.org/10.1016/j.msea.2017.04.052

10. S. Herbst, H.J. Maier, F. Nürnberger, Strategies for the heat treatment of steel-aluminium hybrid components. HTM J. Heat Treatm. Mat. 73(5), 268-282 (2018). https://doi. org/10.3139/105.110368

11. H. Oechsner, Untersuchungen zur Festkörperzerstäubung bei schiefwinkligem Ionenbeschuß polykristalliner Metalloberflächen im Energiebereich um 1 keV. Z. für Phys. 261(1), 37-58 (1973). https://doi.org/10.1007/BF01402280

12. A. Schiffl, W. Fragner, C. Kainhofer, K. Papis, Herausforderungen bei der Präparation von metallischen Hybridwerkstoffen. Prakt. Metallogr. 46(6), 282-291 (2019)

13. G. Timoschenko, Sputtering and secondary electron emission of metals bombarded by argon ions. J. Appl. Phys. 12, 69-77 (1941)

Publisher's Note Springer Nature remains neutral with regard to jurisdictional claims in published maps and institutional affiliations. 